\title{
MicRotúbUlOS Y TERAPIA NEURAL: PROPUESTA DE UNA INVESTIGACIÓN PROMISORIA
}

\author{
Yamile Cruz, MD .', Ramón Fayad, Físico, Ph.D ${ }^{2^{*}}$ \\ ${ }^{1}$ Maestría en Medicina Alternativa-Terapia Neural, Universidad Nacional de Colombia; \\ ${ }_{2}^{2}$ Profesor Titular, Facultad de Ciencias Naturales y Matemáticas, Universidad del Rosario, Bogotá,D.C., Colombia
}

\begin{abstract}
Resumen
La terapia neural es una práctica médica no convencional en la cual se inyectan anestésicos locales (procaína o lidocaína) en concentraciones en un rango de $1-10 \mathrm{mg} / \mathrm{ml}$, en cantidades entre $1-10 \mathrm{~cm}^{3}$, en diferentes partes del cuerpo con fines terapéuticos mas no anestésicos. El mecanismo de acción de los anestésicos locales en esta terapia no se conoce. La posible acción remota de la procaína, el efecto de sus propiedades eléctricas en el transporte de señales en el sistema nervioso y en el restablecimiento del potencial fisiológico de membrana son temas centrales de la terapia neural que requieren una interpretación físico-química. En este artículo proponemos que la propagación de señales en microtúbulos neuronales podría estar involucrada en los mecanismos de acción de la procaína en terapia neural. Sugerimos algunos estudios experimentales que conduzcan a una interpretación científica rigurosa de las observaciones reportadas y de los efectos remotos que se atribuyen a la inyección de procaína en terapia neural.
\end{abstract}

Palabras clave: terapia neural, microtúbulos, procaína, permitividad eléctrica

\section{Microtubules and NeUral therapy: PROPOSAL OF A PROMISSORY RESEARCH}

\begin{abstract}
Neural therapy is a non-conventional medical practice in which the injection of local anesthetics (procaine and lidocaine) in concentrations in the range of $1-10 \mathrm{mg} / \mathrm{ml}$, in quantities between $1-10 \mathrm{~cm}^{3}$, is applied in different parts of the body with therapeutical but not anesthetics purposes. The mechanism of action of local anesthetics in this therapy is not known. The possible remote action and the electrical effect of procaine on the signal transport and on the membrane is normal potential recovery are very important issues in neural therapy, and a physico-chemical explanation of these phenomena is required. In this paper we propose that the propagation of signals in neural microtubules could be involved in the mechanism of action of procaine in neural therapy. We suggest some experimental studies in order to find a more rigorous scientific interpretation of the reported observations and of the remote effects that are assigned to the injection of procaine in neural therapy.
\end{abstract}

Key words: neural therapy, microtubules, procaine, electrical permitivity

\section{Microtúbulos E TERAPIA NEURAL: UMA PROPOSTA DE PESQUISA PROMISSORA}

\begin{abstract}
Resumo
A terapia neural é uma prática médica não convencional em que se injetam anestésicos locais (procaína ou lidocaína) em concentrações variando de 1 a $10 \mathrm{mg} / \mathrm{ml}$, em quantidades 1-10 cm3, em diferentes partes do corpo para fins terapêuticos, mas não anestésicos. O mecanismo de ação dos anestésicos locais nesta terapia não é conhecido. O possível ação
\end{abstract}

Recibido: Abril 26 de 2011 Aceptado: Mayo 11 de 2011

* Correspondencia: Ramón Fayad ramon.fayad@urosario.edu.co, ramon.fayad@gmail.com. Dirección postal: Facultad de Ciencias Naturales y Matemáticas, Universidad del Rosario, Carrera 24 No. 63C-69 Bogotá, Colombia. 
remota da procaína, o efeito das propriedades elétricas no transporte de sinais no sistema nervoso e restabelecimento do potencial fisiológico de membrana são os temas centrais da terapia neural que requerem uma interpretação físico química. Neste artigo propomos que a propagação de sinais em microtúbulos neuronais poderiam estar envolvidos nos mecanismos de ação da procaína na terapia neural. Se sugerem estudos experimentais que levam a uma interpretação científica rigorosa das observações relatadas e dos efeitos remotos que são atribuídas à injeção de procaína na terapia neural.

Palavras-chave: terapia neural, microtúbulos, procaína, permissividade elétrica

\section{Introducción}

La terapia neural es una práctica médica no convencional que ha venido consolidándose en las últimas décadas en el mundo occidental. Las observaciones sobre resultados exitosos de la aplicación de anestésicos locales como lidocaína y particularmente procaína, en soluciones acuosas que no exceden el $1 \%$, en concentraciones en el rango de 1 a $10 \mathrm{mg} / \mathrm{ml}$, y en cantidades que varían entre $1-10 \mathrm{~cm}^{3}$, han recibido particular atención. Estos valores están por debajo de los utilizados con fines anestésicos, pues en terapia neural el propósito de inyectar estos fármacos tiene fines terapéuticos. Los puntos de partida de esta práctica se han basado en observaciones empíricas y está ausente una formulación teórica más completa acompañada de un sustento experimental que explique fenómenos que los neuroterapeutas reclaman como hipótesis validadas, tales como el efecto que tiene la procaína en las membranas de las células enfermas y la acción remota de este fármaco sobre la repolarización de las membranas de estas células al aplicarse en sitios que el neuroterapeuta asume como causantes de patologías. Estos efectos implican la transmisión de señales en el sistema nervioso. En neurociencia clásica se acepta que los trabajos de Hodgkin y Huxley (1) sentaron las bases para analizar las características de los potenciales de acción y estudiar el efecto de sustancias que al actuar sobre los canales iónicos impiden la generación y propagación del impulso nervioso. Los anestésicos locales son unas de estas sustancias. Si bien su mecanismo de acción no es del todo conocido, hay suficiente evidencia que demuestra que inhiben el ingreso de sodio $\left(\mathrm{Na}^{+}\right)$al citoplasma por lo que no se alcanza el valor umbral de despolarización y no se genera el potencial de acción (2).

En las últimas décadas se ha sugerido que la transmisión y procesamientos de información pueden tener lugar a nivel subneuronal. Las aproximaciones al fenómeno han surgido desde el punto de vista de la comprensión de los mecanismos de comunicación en los seres vivos y al surgimiento de nanotecnologías de amplia utilización en la época contemporánea. Los microtúbulos (MT) son estruc- turas fundamentales de las células eucariotas que han sido tema de estudio en este sentido. Algunos mecanismos por los cuales los MT organizan procesos dinámicos intracelulares se conocen y a partir de sus propiedades físicas y químicas se ha sugerido que juegan un papel importante en el transporte de varios tipos de señales (3-5). Se han construido modelos físicos que demuestran que la estructura y composición de los MT les permite transmitir información de un lugar a otro en la misma célula, entre células vecinas o de la célula al medio que la rodea, bien en forma de solitones, o como desplazamiento de electrones (5-8). Se sabe que la propagación de señales en los MT depende de las propiedades eléctricas del medio en que están inmersos, particularmente de la permitividad, pero no hay estudios sistemáticos sobre la alteración de esta propiedad por la inclusión de anestésicos locales en el medio. Algunos resultados revelan que la presencia de anestésicos locales en el citosol disminuye la densidad de los MT en la fibra y también disminuye el transporte axónico (9). En otros casos no hay modificación en la densidad de los MT pero sí se observa disminución en el transporte axónico (10).

Estas observaciones abren la posibilidad de plantear correlaciones formales entre los efectos atribuidos a la procaína en terapia neural y los modelos físicos que explican la conducción de señales en los MT. En este artículo se sugieren algunos estudios que podrían adelantarse para contribuir a una interpretación científica más rigurosa sobre el efecto de la procaína como se emplea en terapia neural. Con el propósito de facilitar la comprensión de la propuesta se presentan someramente los principios de la terapia neural, se revisan algunos mecanismos de los anestésicos locales y se describen dos modelos físicos, uno clásico y otro cuántico, que explican el transporte de solitones y electrones en los MT.

\section{Terapia neural}

La terapia neural es una práctica médica que se inició en Europa a mediados del siglo XIX a partir de las observaciones empíricas reportadas en Rusia por Pavlov, Speransky, 
Vischevsky y Bikov, en Alemania por Spiess, Leriche y Schleich y en Inglaterra por Head. En las primeras décadas del siglo XX, Ferdinand y Walter Huneke propusieron varias hipótesis en las que se basan los esquemas a seguir en esta terapia como se practica en la actualidad, y acuñaron gran parte del léxico que se emplea para expresar los conceptos de enfermedad, salud y el entorno que rodea al individuo como ser social $(11,12)$.

En el texto clásico de Dosch (13) se describen los aspectos más relevantes de los fundamentos de la terapia neural, se presenta el concepto del denominado campo interferente (o irritación) con el que los neuroterapeutas designan a las regiones de tejido en que se encuentran células alejadas del estado fisiológico desde el punto de vista electroquímico, patología que atribuyen a alteraciones en el mecanismo de las bombas de sodio $\left(\mathrm{Na}^{+}\right)$y potasio $\left(\mathrm{K}^{+}\right)$. En el texto se describe el trabajo original de Huneke sobre los "efectos remotos de los anestésicos locales", se citan varias observaciones experimentales en animales y humanos para sustentar que la procaína, aplicada en sitios considerados clave de acuerdo con patologías recientes o anteriores, a las que denominan memorias perniciosas, actúa sobre las células enfermas y les restituye el potencial que deben tener en condiciones normales. Los efectos de la procaína sobre el trofismo y la observación de que altera algunas patologías se encuentran el libro de Fischer (14).

Como el punto de partida en la terapia neural es utilizar procaína con fines terapéuticos y no anestésicos, su empleo se hace a partir de soluciones acuosas, en concentraciones y dosis que están por debajo de los utilizados como anestésico local. La aplicación se hace mediante inyecciones con agujas, cantidades y técnicas variables, que pueden ser pápulas intradérmicas, puntos profundos en troncales o ganglios nerviosos $(15,16)$. La dosis y los lugares de aplicación los escoge el terapeuta neural, en las regiones que sospeche que son los causantes de los desequilibrios, de acuerdo con la "historia de vida" del paciente, en la que se presta particular atención a alteraciones, intervenciones quirúrgicas, cicatrices, antecedentes odontológicos y enfermedades anteriores a la aparición de la patología actual. Su práctica no se basa en los métodos convencionales de estudios de doble ciego puesto que, para el terapeuta neural, cada individuo debe tratarse de una manera diferente con base en que la historia de cada evento traumático también lo es.

Este es uno de los argumentos utilizados en la terapia neural para explicar por qué la reacción de cada paciente a la aplicación de procaína durante la terapia no es la misma $(11,14,15)$ pues, según los neuroterapeutas, el equilibrio fisiológico, al que suelen referirse como el orden, es propio de cada persona. En un esfuerzo por comprender un poco más los puntos de partida de estas concepciones vale acudir a algunas aproximaciones epistemológicas a fenómenos de organización, conservación y perpetuación en sistemas biológicos que se encuentran en las referencias $(12,17-19)$ y que los autores de documentos sobre terapia neural citan con frecuencia.

Los neuroterapeutas afirman que en estados de debilitamiento o enfermedad la célula pierde la capacidad de responder a los estímulos, permanece despolarizada y la procaína, por su potencial eléctrico, al que le asignan un valor de $290 \mathrm{mV}$, produce un efecto que permite la repolarización de la membrana de la célula enferma y se recupera la función fisiológica normal. En los textos de terapia neural no se describe con claridad el método utilizado ni las condiciones para determinar este valor. Desde la metodología de las ciencias naturales y de la medicina alopática no es clara la relación de causalidad entre el valor del potencial eléctrico de la procaína y el efecto que, según se reclama, repolariza células muy distantes del lugar de aplicación de este fármaco en bajas concentraciones y en pequeñas dosis $(14,15,19,20)$.

Aún en ausencia de explicaciones causales, los resultados de la terapia neural se reconocen, es una práctica ejercida por médicos y se ha ido consolidando como lo que se denomina, a veces indistintamente, medicina alternativa o complementaria. Por tanto, es de interés adelantar diseños experimentales que permitan una construcción teórica que sugiera explicaciones a las observaciones terapéuticas que se reportan.

\section{Anestésicos locales}

Con el propósito de establecer relaciones entre las propiedades de la procaína y la transmisión de señales a nivel subneuronal, es conveniente presentar comentarios básicos sobre los anestésicos locales y mencionar algunos efectos de la presencia de procaína sobre la densidad de los MT en las neuronas.

Los trabajos de Meyer y Overton sobre la correlación entre el poder de los anestésicos y la solubilidad, particularmente en medios lipídicos, se han discutido recientemente (21). Se sabe que los anestésicos inhiben la permeabilidad al $\mathrm{Na}^{+}$, de manera que no se genera el potencial de acción. En contraposición a la propuesta de Franks y Lieb (22), en el sentido de que la presencia de moléculas en el bolsillo hidrofóbico es suficiente para que se considere anestesia, 
se han reportado observaciones en las que se muestra que algunas sustancias que no tienen efectos anestésicos se fijan a los mismos sectores hidrofóbicos al que se unen las que sí lo tienen (23). Estas observaciones indican que no se dispone de teorías y resultados experimentales suficientes que expliquen satisfactoriamente el mecanismo de acción de los anestésicos.

El descubrimiento de los anestésicos locales se atribuye a Freud y Koller $(24,25)$ quienes realizaron los primeros estudios sobre los efectos farmacológicos de la cocaína, un alcaloide extraído de la planta Erythroxylon coca. Su utilización no tiene como propósito actuar sobre el sistema nervioso central y aplicados en dosis clínicamente establecidas no producen daños en las células nerviosas, pero en dosis muy elevadas pueden producir efectos tóxicos en los vasos sanguíneos $(21,26)$. Los amino-ésteres, como la procaína, se metabolizan por colinesterasas plasmáticas y los metabolitos resultantes permanecen activos por unas horas sin acciones anestésicas hasta que se eliminan(2).

Se ha demostrado que los anestésicos locales, en general, se unen al segmento S6 ubicado en la unidad $\alpha$ del canal de $\mathrm{Na}^{+}(27)$ como se ha descrito en la revisión realizada por Scholz (28). El bloqueo de los canales de $\mathrm{Na}^{+}$por las fracciones apolares de los anestésicos locales no depende de si la entrada externa del canal (29) se encuentra abierta o cerrada (29). La forma no ionizada del anestésico actúa como vehículo para atravesar la fase lipídica de la membrana de la célula nerviosa, mientras la fracción anestésica actúa en el sector del canal en el interior de la célula cuando se encuentra abierto $(24,29,30)$. La fracción ionizada como catión, que en el caso de la procaína es el 98\% (31), es la responsable de la interacción con el receptor y por tanto lo es de la actividad farmacológica $(26,32)$. Sin embargo, varios estudios experimentales indican que la fracción no ionizada también actúa como anestésico y debe dársele importancia en la efectividad de la procaína como anestésico local (25).

Puesto que en el sistema nervioso periférico los canales de $\mathrm{K}^{+}$contribuyen a la fase de repolarización del potencial de acción y juegan un papel central en el restablecimiento del potencial de reposo (1), es de interés mencionar que los anestésicos locales también bloquean los canales de $\mathrm{K}^{+}$ dependientes de voltaje (28). La mayor diferencia entre el efecto de los anestésicos locales sobre los canales de $\mathrm{Na}^{+}$ y los de $\mathrm{K}^{+}$es su baja afinidad por estos últimos. Desde el punto de vista de la terapia neural este hecho debería considerarse como un elemento importante en una de sus hipótesis fundamentales, pues para el neuroterapeuta la célula es una pequeña batería de $\mathrm{K}^{+}$cuyo potencial de reposo está en el rango de $-40 \mathrm{mV}$ a $-90 \mathrm{mV}$ y funciona solamente si permanece en condiciones de recibir iones $\mathrm{K}^{+}$en forma constante (20). Es de notar que estos valores están en los rangos del potencial de equilibrio del $\mathrm{K}^{+}$.

En estudios en el nervio ciático de conejo y en la corteza cerebral de rata, se encontró que la procaína interactúa con las proteínas de los MT reduciendo la densidad de éstos en los axones $(9,10)$. Otros trabajos revelaron que los anestésicos locales también disminuyen la densidad de los MT en los axones sin mielina (33). Hay evidencias de que la presencia de anestésicos locales en fibroblastos disminuye la secreción de colágeno, efecto que se atribuye a modificaciones en la estructura de los $\operatorname{MT}(26,34)$. Es interesante mencionar que una toxina como lo es la tetrodotoxina (TTX), por su efecto inhibidor sobre el movimiento de $\mathrm{Na}^{+}$a través de la membrana, produce efectos como anestésico local (32) y como analgésico de efecto sistémico (35-38).

En los estudios citados se encuentra que la presencia de algunos anestésicos locales en el citosol no altera la densidad de los MT pero sí hay modificación en el transporte axónico. De estos resultados no es posible encontrar una correlación significativa entre la modificación en la densidad de los MT y el transporte axónico, en particular la conducción de señales. Los datos de que se dispone no son suficientes para atribuir la disminución o posible amortiguamiento del transporte de señales en los axones solamente a los MT. Se requieren estudios más completos pues en el fenómeno de conducción de señales en el sistema nervioso el contenido de trifosfato de adenosina (ATP) juega un papel muy importante.

\section{Estructura de los microtúbulos y trasporte de señales}

Para plantear correlaciones posibles entre las propiedades eléctricas de la procaína y su efecto en la conducción de señales a nivel subneuronal, es ilustrativo presentar algunas aproximaciones físicas que describen cómo se propagan señales ondulatorias y electrones libres en los MT.

\section{Estructura de los microtúbulos}

El interior de las células eucariotas contiene una variedad de estructuras fundamentales compuestas por polímeros de proteínas. Junto con los microfilamentos y los filamentos intermedios, los MT forman parte del citoesqueleto a los que se les atribuyen funciones como la movilidad celular, la mitosis, el transporte axónico y la conducción de señales (6-8). Algunos estudios reportan que los MT son elementos 
clave en los inicios de cambios patológicos a nivel celular, como la enfermedad de Alzheimer (39) y ciertos tipos de cáncer (40). Las propuestas de Hameroff $(3,41)$ y Penrose (42) le asignan a los MT participación en el surgimiento y mantenimiento de la conciencia.

La estructura de los MT y los mecanismos involucrados en su ensamblaje y desensamble están bien establecidos (4,4347). Las unidades estructurales son dímeros que se forman por la unión de tubulina $\alpha$ y tubulina $\beta$. Los dímeros se ensamblan en forma de cadenas que se denominan protofilamentos, que se agregan lateralmente y forman cilindros huecos cuyos diámetros internos y externos son $15 \mathrm{~nm}$ y $25 \mathrm{~nm}$, respectivamente (47-49). La longitud de un dímero es de unos $8 \mathrm{~nm}$ y la de los protofilamentos está en el rango $1 \mu \mathrm{m}-100 \mu \mathrm{m}$, aun cuando se han reportado valores hasta de $1 \mathrm{~m}$ en algunos mamíferos $(39,41,47)$. En un MT típico hay trece protofilamentos. Durante la polimerización ambas unidades de tubulina se encuentran unidas a una molécula de guanosín trifosfato (GTP), que desempeña una función estructural en la tubulina $\alpha$ pero es hidrolizada a guanosín difosfato (GDP) en la tubulina $\beta$. La Figura 1 muestra un esquema simplificado que describe los pasos que tienen lugar en el ensamblaje de un MT.

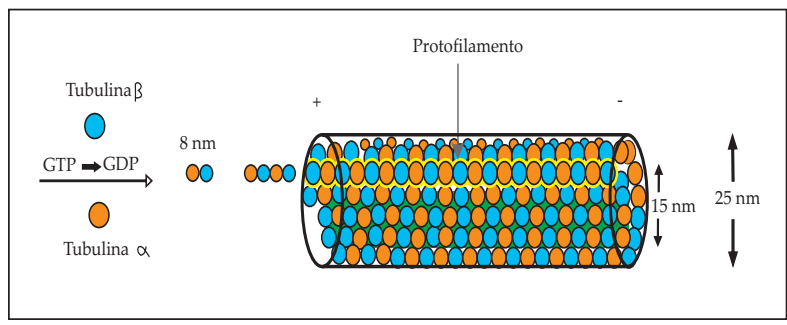

Figura 1. Esquema simplificado que ilustra los pasos en el ensamble de un microtúbulo.

La hidrólisis de GTP a GDP libera $0.21 \mathrm{eV}$ de energía $(45,48,49)$ y parte de la energía de este proceso modula la adición de nuevos dímeros a los protofilamentos que se han formado, pero aun no se comprende por qué no se utiliza la totalidad de la energía en el fenómeno de adición de dímeros. Se ha propuesto que la fracción de energía disponible cambia la rigidez de los enlaces o contribuye a la conformación de redes que facilitan la generación de excitaciones coherentes (8). Una característica muy importante de los MT es su polaridad eléctrica. Como la adición de dímeros es la unión de "cabeza" con "cola" para formar protofilamentos, las filas sesgadas en la superficie de monómeros de tubulina $\alpha$ y tubulina $\beta$ originan una polaridad global en el MT.
El extremo compuesto por una anillo de tubulina $\alpha$ se denomina extremo (-) y el opuesto, por tubulina $\beta$, extremo $(+)$. Los MT no se mantienen permanentemente, cambian en número y longitud durante la diferenciación celular (50) y su tiempo de vida es de 20 horas aproximadamente (51). Las proteínas asociadas a los MT, designadas como $\mathrm{MAP}$, son de particular importancia en el proceso de ensamblaje. Se ha demostrado que su presencia determina la estabilidad del crecimiento de la estructura de los cilindros y que el extremo $(+)$ crece más rápido que el terminal (-) (52-54). En el interior de los cilindros y en el medio que los rodea se encuentran moléculas de agua de manera que existe un gran momento dipolar eléctrico y se genera un campo eléctrico intrínseco paralelo al eje del cilindro. Las evidencias sugieren que las propiedades electromagnéticas de la célula, en este caso la polaridad de los MT, son las responsables de que estas estructuras no estén orientadas en forma azarosa dentro de los axones.

En la literatura se reportan algunos modelos para explicar la organización ordenada de los MT en células neuronales (6-8). Parece ser que las interconexiones e intraconexiones que realizan las MAP facilitan el proceso de transporte axónico. Un caso de particular interés es el de la MAP denominada tau $(\tau)$; su presencia, que aumenta con la distancia al soma, incrementa la estabilidad de la estructura y juega un papel importante en las propiedades de transferencia de señales, en el aprendizaje y en la memoria $(26,55)$.

\section{Transporte de señales en los MT}

Las observaciones experimentales muestran que los MT son estructuras altamente organizadas que exhiben propiedades piezoeléctricas y piroeléctricas (56). La aplicación de un campo eléctrico intenso a lo largo del MT tiene como efecto que los dipolos se orienten en la dirección del campo. Cuando el campo cesa, los dímeros pueden regresar a su configuración inicial y liberar la energía almacenada. Hace varias décadas Fröhlich $(57,58)$ propuso que las características de los sistemas biológicos, por sus propiedades dieléctricas, con posibilidades de que sus componentes posean actividad vibracional dipolar y por las variadas regiones en las que se encuentran electrones libres, sugieren la posibilidad de encontrar estados de coherencia en la propagación de señales que viajan sin que haya disipación de energía. Los denominados condensados de Fröhlich son similares a los de Bose-Einstein; son sistemas con la propiedad colectiva de coherencia cuántica macroscópica, de manera que distintos osciladores pueden lograr un estado que vibre en resonancia. 


\section{Transporte de solitones en los MT: un modelo clásico}

Las aproximaciones desde la física clásica al transporte de señales en los MT asumen que los dímeros pueden estar en la conformación $\alpha$ o en la $\beta$ y que cada dímero está influenciado por sus dímeros vecinos. Se pueden construir modelos no lineales que justifiquen la transferencia de señales ondulatorias y establezcan marcos conceptuales entre el exceso de energía que se libera en la hidrólisis de GTP después del ensamble de la tubulina y las funciones que se le atribuyen a los MT (59). Sus propiedades piezoeléctricas sugieren interpretaciones entre la estructura interna de los axones y su función principal como es la comunicación sináptica.

Con la formulación de Hamiltonianos, que incluyen los factores más relevantes de las interacciones de los componentes de los MT con el medio en que están inmersos, Chou et al. $(6,7)$ demostraron que parte de la energía que se libera en la hidrólisis de GTP viaja a lo largo de los MT en forma de pulsos excitados. Suponiendo que los dímeros de tubulina poseen momentos dipolares que se ordenan espontáneamente a temperatura fisiológica, Sataric et al.(6), Trpisova et al. $(7,60)$ y Tuszynski et al. $(8,61)$ elaboraron modelos que demuestran que esa fracción de energía viaja a lo largo del protofilamento en forma de onda solitaria (denominada solitón). Los aspectos más relevantes y las conclusiones que se derivan de estos modelos se presentan a continuación.

Las propuestas se basan en las demostraciones experimentales de Athenstaedt et al. (62) quienes reportaron cambios conformacionales inducidos por la hidrólisis de GTP, en donde un monómero desplaza su orientación del eje vertical; supusieron que el único grado de libertad está relacionado con la proyección del desplazamiento del monómero de su posición de equilibrio sobre el eje del cilindro del MT. Este desplazamiento causa una anarmonicidad cristalina que se representa por un potencial de cuarto orden, tema que ha sido bastante estudiado para dipolos en cristales ferroeléctricos (63) cuyas propiedades también se presentan en los MT. Con estas consideraciones, el Hamiltoniano para la dinámica de los dipolos en un MT es

$$
H=\sum_{n}^{N}=1\left[\frac{1}{2} M\left(\frac{d u_{n}}{d t}\right)^{2}\right]+\frac{1}{2} K\left(u_{n+1}-u_{n}\right)^{2}-\frac{A}{2} u_{n}^{2}+\frac{B}{4} u_{n}^{4}-q E u_{n}
$$

donde $u_{n}$ representa la proyección del desplazamiento longitudinal del n-ésimo dímero sobre todo el protofilamento, $N$ es la cantidad de dímeros y $M$ es la masa de cada dímero. El primer término es la energía cinética, el segundo la energía elástica dada por las vibraciones de los dímeros cercanos, el tercer y cuarto términos representan el potencial anarmónico y el último $-q E u_{n}$ es la energía eléctrica debida al campo eléctrico externo al MT. Las cantidades A y B son constantes, E es el campo y q es la carga móvil en el protofilamento.

Como los MT están inmersos en el citosol, que es una solución líquida de iones que interactúa con el MT tanto por las fuerzas eléctricas como por la fuerza viscosa que amortigua el desplazamiento en los dímeros de tubulina, estos efectos deben incluirse en la ecuación de movimiento. Después de un extenso procedimiento matemático, en el límite continuo, se llega a una de las expresiones de la ecuación de sine-Gordon, cuyas soluciones son ondas tipo solitón que se propagan a lo largo del MT. Trpisova et al. (60) estimaron que la velocidad de propagación del solitón es del orden de $10^{-2} \mathrm{~m} / \mathrm{s}$. En el mismo trabajo, Tripsova et al.(60) propusieron otro modelo con base en la polarización en los MT y para esta simulación la velocidad de propagación de la onda es muchísimo mayor, pues determinaron un valor del orden de $10^{2} \mathrm{~m} / \mathrm{s}$. En estos dos modelos se asumió un valor de 70 para la permitividad relativa del citosol.

Es de resaltar la dependencia de la velocidad de propagación con la intensidad del campo eléctrico externo. La energía adicional suministrada por este campo es la que justifica la creación de las excitaciones tipo kink que se mantienen para que se transporte la información. La intensidad del campo puede disminuirse en varios órdenes de magnitud si se aumenta considerablemente la permitividad eléctrica del medio en el que se encuentran los MT. Los datos de Román et al. (64) indican que, en muestras tomadas en condiciones de anestésico o con fines terapéuticos, la permitividad eléctrica de la procaína, está entre $4 \times 10^{6}$ y $1,2 \times 10^{7}$. Si se considera que la procaína ingresa al citosol y si se analizan algunas posibles geometrías de esta situación, puede sugerirse que la presencia de este fármaco debe modificar sustancialmente las interacciones eléctricas entre el citosol y los MT, de manera que algunas propiedades de la propagación de señales se alteran. Para ese valor de permitividad se trata de un condensador con enorme capacidad para almacenar cargas eléctricas. Quedaría por interpretarse el significado físico y biológico del efecto de la procaína en las características eléctricas del citosol, en las de los MT y en el potencial de membrana.

\section{Trasporte de electrones en los MT: un modelo cuántico}

Los modelos cuánticos asumen que cada dímero se encuentra en un estado que es la superposición de las conformaciones $\alpha$ y $\beta$ y presuponen que existen estados 
coherentes entre las cadenas de dímeros. La formulación de Hamiltonianos que incluyen el salto de electrones entre los dímeros de tubulina y entre dos dímeros consecutivos ha demostrado que podrían presentarse estados de coherencia cuántica que, de mantenerse por un tiempo lo suficientemente largo, permitirían que tenga lugar la propagación de señales sin disipación de energía. Las conjeturas principales están en considerar que el interior del MT es una cavidad y no una guía de ondas.

En un trabajo de Tuszynski et al. (8) se reportaron unas propiedades que sugieren que los MT participan en la conducción de señales eléctricas dentro de la célula vía mecanismos de transferencia de electrones que pueden estar acoplados con la hidrólisis de GTP. Con base en la estructura y composición de los MT, se supone que para cada dímero de tubulina hay dos electrones que pueden moverse a lo largo del protofilamento. Al calcular la cantidad de electrones que pueden viajar y con la determinación de la conductividad del protofilamento se encuentra que estas propiedades son similares a las que se presentan en materiales semiconductores.

Tuszynski et al. (61) construyeron un modelo en el que cada protofilamento se representa como una cadena de potenciales y el electrón se propaga enfrentando un potencial de doble pozo cuadrado por cada dímero y por cada par de dímeros consecutivos. La Figura 2, tomada de Martínez et al. (65), que es una modificación de la originalmente publicada por Tuszynski et al. (8), ilustra el pozo de potencial en el que las barreras de energía que se proponen para el electrón que viaja son $0,4 \mathrm{eV}$ para saltos en el interior del dímero y de $1,0 \mathrm{eV}$ para el salto entre dos dímeros consecutivos. La separación entre los electrones en la tubulina es de 2,0 nm y 0,7 nm, y las energías potenciales entre dos electrones separados por estas distancias son $2,0 \mathrm{eV}$ y $0,7 \mathrm{eV}$, respectivamente.

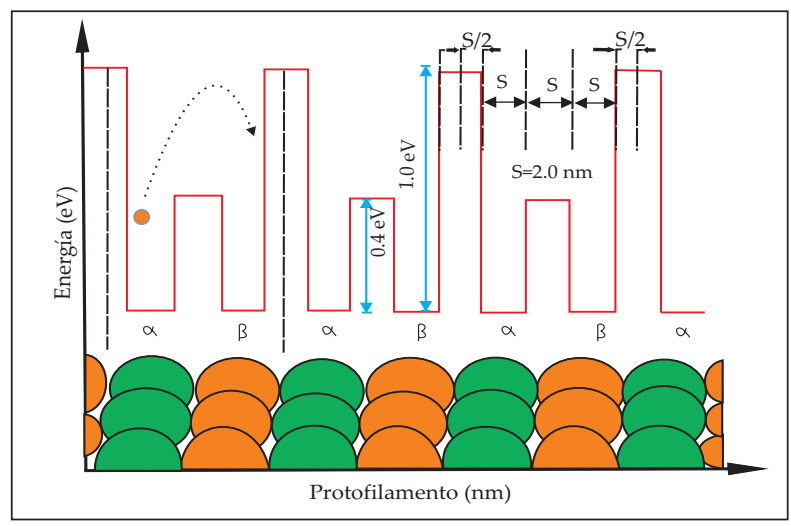

Figura 2. Barreras de potencial que enfrenta un electrón que viaja a lo largo de un protofilamento en un microtúbulo neuronal (Tomado de (68)).
Martínez et al. (65) adecuaron los trabajos de Tuszynski et al. (8), calcularon el número de dímeros que recorre el electrón para energías del orden de 2,0 eV y encontraron que cuando el coeficiente de transmisión es 0,075 el electrón recorre 1250 dímeros. Se concluye que con este modelo y para este valor de energía una señal eléctrica en forma de transporte de electrones puede viajar a lo largo de todo el protofilamento (66). Estas aproximaciones cuánticas se han utilizado recientemente para demostrar que a lo largo de los MT, además de solitones y señales eléctricas, también viajan fotones (67). Para el caso de sistemas biológicos, las suposiciones anteriores se han sustentado en que las tubulinas están ordenadas geométricamente en forma de redes en los MT.

Para aproximarse al efecto de anestésicos sobre la propagación de señales en los MT, se ha sugerido que las oscilaciones de los dipolos dentro de los bolsillos hidrofóbicos están involucradas en la coherencia macroscópica de manera que los procesos cuánticos que tienen lugar en esa región se inhiben por estos fármacos y debilitan la movilidad de los electrones al alterar las fuerzas de London y van der Waals $(27,57,58)$. Se ha especulado, incluso, que hay dependencia de la conciencia de los estados cuánticos de los electrones dentro de los bolsillos hidrofóbicos en cierta clase de proteínas cerebrales $(3,42)$ pero este tema no cabe en los propósitos de este artículo.

Aun cuando la investigación experimental in vivo no ha reportado en forma indiscutible los condensados de Fröhlich, en la literatura se encuentran diseños factibles para que, al menos in vitro, se produzcan estados coherentes de Bose-Einstein y Fröhlich en oligómeros policristalinos a temperaturas fisiológicas (68). En un trabajo muy reciente se demuestra que en algunos sistemas biológicos se presentan casos explícitos de coherencia cuántica, que permanecen durante tiempos medibles y que los hace observables, como ocurre en los procesos de información en el cerebro de una especie de aves (69).

La controversia sobre la presencia de estados de coherencia cuántica en sistemas biológicos se manifiesta cuando a los MT se les atribuyen propiedades en los que se involucran procesos de pensamiento y conciencia (70), mas no cuando la evidencia experimental sugiere que estas estructuras están involucradas en fenómenos de aprendizaje y memoria $(26,55)$. Puesto que los MT están relacionados con el transporte axónico, es posible proponer que la propagación de señales está involucrada en la actividad eléctrica de las células excitables. De los modelos que se presentan en este artículo y de resultados que se reportan en las referencias citadas es clara la importancia 
de adelantar estudios orientados a encontrar el significado biológico de la propagación de señales en MT y su papel en el procesamiento de información eléctrica dentro del axón.

\section{Discusión}

El sustento de la terapia neural con observaciones empíricas es reconocido. Sin embargo, los puntos de partida epistemológicos en los que se basa, la atribución a patologías causadas por lo que los neuroterapeutas denominan irritaciones o campos interferentes y el papel terapéutico de la procaína, entre otras cosas, no han sido dilucidados. En esfuerzos por interpretar los comportamientos observados, desde hace varias décadas algunos médicos dedicados e esta práctica (71) sugieren abordar el tema desde las teorías de los sistemas dinámicos, los fenómenos caóticos, los sistemas abiertos y los principios de la mecánica cuántica. Sin embargo, no se encuentran resultados de estas aproximaciones y su relación con la terapia neural que se hayan sometido a la comunidad científica por medio de publicaciones en revistas de amplia circulación internacional. Debe anotarse que la utilización de varios términos que son parte del léxico propio de la terapia neural tiene fundamento en las raíces etimológicas, pero en algunas ocasiones no corresponden a los empleados comúnmente en ciencias naturales y no es fácil encontrar analogías en sus significados, lo que no implica que deben encontrarse nuevas leyes o que se trate de excluir las manifestaciones de los organismos vivos de las leyes que rigen el comportamiento físico de la naturaleza. Quizás se necesite identificar algunas propiedades emergentes en biología para aclarar su comportamiento con los marcos teóricos científicamente aceptados.

Con el propósito de determinar el efecto de la procaína en la conducción de señales eléctricas en los MT y establecer alguna relación con las propiedades que le asignan a ese fármaco en terapia neural, se propone un experimento análogo a los descritos por Priel et al. $(72,73)$. En uno de estos estudios (72) se encontró que en tubulinas polimerizadas en presencia de taxol, los MT, en su interior, se comportan como transistores biomoleculares, que son amplificadores en el sentido de que al aplicar un estímulo eléctrico en uno de sus extremos, en el otro se detecta una señal eléctrica 300 veces mayor que el estímulo aplicado. De los resultados se concluye, además, que los MT mejoran la conectividad eléctrica entre dos lugares en solución salina y que los fenómenos se detectan en los dos sentidos a lo largo de los MT. Estos datos se han complementado con observaciones recientes, según lo reporta Etienne-Manneville (74).

Si en lugar de taxol se utiliza procaína, es posible hacer mediciones como las reportadas por Priel et al. (72) con

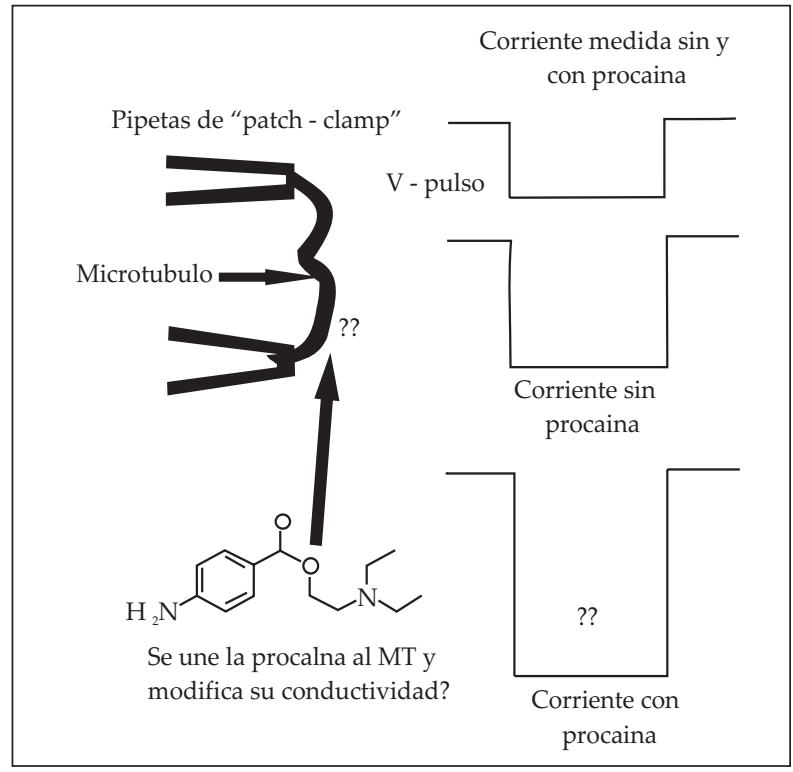

Figura 3. Propuesta experimental para determinar el efecto de la procaína en las propiedades eléctricas de los MT.

un diseño experimental como el que se muestra en la Figura 3. En esta propuesta, se atrapa el MT entre dos pipetas de "patch" y se mide su conductividad eléctrica. Para establecer comparaciones, a partir de este resultado se aplican distintas concentraciones de procaína al medio y se repiten las medidas. Si la procaína afecta la conducción iónica o electrónica la diferencia se apreciará como un cambio en la conductividad que mide una de las pipetas conectada a un amplificador FET (amplificador con transistores de efecto de campo).

Con los resultados que se obtengan podrán sugerirse interpretaciones teóricas sobre la distribución de cargas entre el medio interior y el exterior en los MT, de manera que, dada la alta permitividad de la procaína, podría inferirse que en el medio externo se crearía una nube iónica adyacente al MT que permitiría la amplificación de señales que se propagan a lo largo del eje del MT. Esta amplificación, de llegar a observarse, suministraría información sobre el proceso de transmisión eléctrica en los MT y su acoplamiento con la función de los canales iónicos. Este sería un resultado importante para interpretar la comunicación entre microtúbulos y la transmisión sináptica, además de que podría servir de base para explicar el efecto de las "microdosis" de procaína en neuronas.

Los MT en las dendritas están dispuestos en forma de redes de polaridad mixta interconectados por proteínas MAP2. Si una señal se inicia en un extremo del MT viaja a lo largo de los protofilamentos, se amplifica y sirve como 
señal de entrada en el siguiente. Si se acepta la hipótesis de Priel et al.(72) de que los MT operan como una gran "máquina de estado", se tendría que la señal de salida de un MT es el estado del sistema que se "lee" para propagar y estimular eléctricamente canales iónicos sensibles al voltaje que se encuentran distantes del punto donde se inició la señal. Es de notar que si de los resultados que se obtengan en el experimento propuesto con procaína en el citosol se encuentra este hecho y además se determina que se trata de un modelo de entradas y salidas, en donde las salidas dependen no solamente de las señales de entrada actuales sino también de las anteriores, podrían sentarse las bases para interpretar la acción remota de la procaína y aproximarse, de alguna manera teórica y aun incipiente, a lo que en terapia neural se denominan "campos interferentes" $\mathrm{y}$ "memorias perniciosas".

\section{Agradecimientos}

Al doctor José Antonio Ramírez Latorre, Profesor Titular de la Escuela de Medicina y Ciencias de la Salud de la Universidad del Rosario, por la lectura crítica del manuscrito y por su disposición a adelantar el estudio experimental que se propone. Al doctor Julio César Payán de la Roche, Médico, Ginecólogo, Obstetra, Neuroterapista, pionero de la terapia neural en Colombia, por compartir la importancia de reflexionar, escribir y publicar sobre este tema en revistas científicas.

\section{Referencias}

1. Hodgkin AL, Huxley AF. A quantitative description of membrane current and its application to conduction and excitation in nerve. J Physiol. 1952;117:500-44.

2. Becker E, Reed K L. Essentials of local anesthetic pharmacology. Anesth Prog. 2006;53(3):98-108.

3. Hameroff S, Penrose R. Orchestrated reduction of quantum coherence in brain microtubules: A model for consciousness. Math Comput Simulat .1996;40(3-4):453-80.

4. Allen D R. El microtúbulo motor intracelular. Investigación y Ciencia. 1987;127:18-25

5. Satarić M V, Ilić D I, Ralević N,Tuszynski J A. Nonlinear model of ionic wave propagation along microtubules. Eur Biophys J. 2009;38(5):637-47.

6. Sataric M V, Tuszynski J A, Zakula R B. Kinklike excitations as an energy-transfer mechanism in microtubules. Phys Rev E Stat Nonlin Soft Matter Phys.1993;48(1):589-97.

7. Chou K C, Zhang C, Maggiora G. Solitary wave dynamics as a mechanism for explaining the internal motion during microtubule growth. Biopolymer. 1994;34(11):143-53.

8. Tuszynski J A, Brown J A, Hawrylak P. Dielectric polarization, electrical conduction, information processing and quantum computation in microtubules. Are they plausible? Philos Transact A Math Phys Eng Sci. 1998;356:1877-926.

9. Lavoie P A, Khazent T, Filion P R. Mechanisms of the inhibition of fast axonal transport by local anesthetics. Neuropharmacology. 1989;28(2):175-81.
10. Bisby M A. Inhibition of axonal transport in nerves chonically treated with local anesthetics. Exp Neurol. 1975;47:481-9.

11. Payán J C. Lánzate al vacío y se te extenderán tus alas. Colombia: Mc Graw Hill; 2000.

12. Torre M R. Terapia neural: ¿defensores o detractores? [en línea]. 2001 [fecha de acceso 2 de noviembre 2010]; VRL Disponible:http://www. amc.sld.cu/amc/2001/v5n2/Terapia_Neural.htm

13. Dosch P. Libro de la enseñanza de la terapia neural según Huneke: Alemania Heidelberg; 1973.

14. Fischer L. Terapia neural según Huneke fundamentos técnicas, aplicación práctica. México: Hippokrates; 2006.

15. Beltrán E. Terapia Neural, Documento Electrónico Aula Virtual grupo académico de medicina alternativa, Universidad Nacional de Colombia. 2009.

16. Morais A, Gaspar M, Marinho S, Pinto R J. Allergy to local anesthetics of the amide group with tolerance to procaine. Allergy. 2003;58(8):827-8.

17. Solana J, Morín E. Por un pensamiento complejo: implicaciones interdisciplinarias. Madrid: Akal S.A; 2005.

18. Maturana H, Varela F. De máquinas e seres vivos. Autopoiese, a Organização do Vivo. Porto Alegre: Artes Médicas, 1997.

19. Dosch P, Dosch M. Manual of neuratherapy according to Huneke. New York : Thime;2007

20. Barop H. Atlas de terapia neural. México: Hippokrates Verlag Stuttgart: 2003.

21. Olivier C, Wenker MD. Review of currently used inhalation anesthetics: Part I J.Anesthesiol.[en línea],1999. [fecha de acceso 14 de noviembre 2010]

22. Texto online: http://www.ispub.com/ostia/index.php?xmlFilePath= journals/ija/vol3n2/inhal1.xml

23. Franks N P, Lieb $\mathrm{W}$ R. Which molecular targets are most relevant to general anaesthesia? Toxicol Lett .1998;100(101):1-8.

24. Fang Z X, Sonner J, Laster M J. Anesthetic and convulsant properties of aromatic compounds and cycloalkames: implications for mechanisms of narcosis. Anesth Analg. 1996;83(5):1097-104.

25. Smerlilli A S. Anestésicos locales: historia, acción farmacológica, mecanismo de acción, estructura química y reacciones adversas. Rev Fac Odontol Univ Buenos Aires. 2004;19(46):19-24.

26. Yvan A, Ruetsch A, Bönibc T, Alain B. From cocaine to ropivacaine: The history of local anesthetic drugs. Curr Top Med Chem. 2001;1:17582.

27. Miyamoto Y, Muto E, Mashimo T, Iwane AH, Yoshiya I, Yanagida T. Direct inhibition of microtubule-based kinesin motility by local anesthetics. Biophys J. 2000;78(2):940-9.

28. Franks N P, Lieb W R. Do general anesthetics act by competitive binding to specific receptors. Nature. 1984;310:599-601.

29. Scholz A. Mechanisms of (local) anaesthetics on voltage-gated sodium and other ion channels. Br J Anaesth. 2002;(89):52-61.

30. Imbelloni L, Diaz A, Gaspar M, Gouveia A, Cordeiro J. Evaluación de la densidad de los anestésicos locales y de la combinación con adyuvantes. Rev. Bras. Anestesiol. 2009; 59(2):88-93.

31. Whizar V, Flores J, Puerta G. Toxicidad de los anestésicos locales [en línea], 2002. [fecha de acceso 2 de noviembre 2010] VRL.

32. Texto online: www.anestesiadolor.org/anestesia/docs/toxici$\underline{\text { dad de los anestesicos locales.html }}$ 
33. Tucker G T, Mather L. Properties, absortion and disposition of local anesthetics agents: Neural Blockade in clinical anesthesia and management of pain. Philadelphia: Lippincot-Raven; 1998. [fecha de acceso 17 de noviembre 2010].

34. Texto online: http://www.cookcountyregional.com/Chapter2.pdf

35. Marcil J, Walczak JS, Guindon J, Ngoc AH, Lu S, Beaulieu P. Antinociceptive effects of tetrodotoxin (TTX) in rodents. Br J Anaesth. 2006;96(6):761-8.

36. Milos H, Hameroff S, Katheleen M D. Local anesthetics and wound healing. J Surg Res. 1979;27:367-71.

37. Pierzchalska M, Michalik M, Stepień E, Korohoda W. Changes in morphology of human skin fibroblasts induced by local anaesthetics: role of actomyosin contraction. Eur J Pharmacol. 1998; 358(3):235-44.

38. Adams H J, Murray R B, Takman B H. The local anesthetic activity of tetrodotoxin alone and in combination with vasoconstrictors and local anesthetics. Anesth analg. 1976;55(4):568-73.

39. Padera R F, Tse J Y, Bellas E, Kohane D S. Tetrodotoxin for prolonged local anesthesia with minimal myotoxicity. Muscle Nerve. 2006;6(34):743-53.

40. Hagen N, Du S P, Lapointe B, Ong L M, Dubuc B, Walde D, Love R, Ngoc A H. Tetrodotoxin for moderate to severe cancer pain: a randomized, double blind, parallel design multicenter study. J Pain Symptom Manage. 2008;35(9):420-9.

41. Covino B, Giddon D. Pharmacology of local anesthesic agents. J Dent Rev. 1981;60:1454-9.

42. Mandelkow E, Song Y H, Schweers O, Marx A, Mandelkow E M. On the structure of microtubules, tau, and paired helical filaments. Neurobiol Aging. 1995;16(3):347-54.

43. Zhous J, Giannakakous P. Targeting microtubules for cancer chemotherapy. Curr Med Chem Anti-Cancer Agents. 2005;5:65-71.

44. Hameroff S, Watt R C. Information processing in microtubules. J Theor Biol. 1982;98:549-61.

45. Penrose R. Lo grande y lo pequeño de la mente humana. Madrid humana.Madrid: Cambridge; 1999.

46. Conde C, Cáceres A. Microtubule assembly, organization and dynamics in axons and dendrites. Nat Rev Neurosci. 2009;10:319-31.

47. Schaap I A, Carrasco C P, MacKintosh J, Frederick C, Christoph F. Elastic response, buckling, and instability of microtubules under radial indentation. Biophys J. 2006;91(4):1521-31.

48. ayley P, Schilstra M, Martin S A. Lateral cap model of microtubule dynamic instability. FEBS Lett. 1989;259(1):181-4.

49. Faber J, Portugal R, Rosa L P. Information processing in brain microtubules. Biosystems. 2006;83(1):1-9.

50. Kamarova Y. Life cycle of MTs: persistent growth in the cell interior, asymmetric transition frequencies and effestts of the cell boundary. J Cell Sci. 2002;115:3527-39.

51. Insinna E M, Zaborski P, Tuszynski J. Electrodynamics of microtubular motors: the building blocks of a new model. Biosystems. 1996;39(3):187-226.

52. Cassimeris L. Microtubule assembly: lattice GTP to the rescue. Curr Biol. 2009;19(4):174-6.

53. Yu W, Baas, P W. Changes in microtubule number and length during axon differentiation. J Neurosci. 1994;14(5):2818-29.
54. Poulain F E, Sobel A. The microtubule network and neuronal morphogenesis: dynamic and coordinated orchestration through multiple players. Mol Cell Neurosci. 2010;43(1):15-32.

55. Fourest A, Peris L, Gache V, Garcia I, Juillan B, Lantez V, Job D. Microtubule rgulation in mitosis: Tubulin phosphorylation by the cyclin-dependent kinase Cdk1. Mol Biol Cell. 2006;17(3):1041-50.

56. Mandelkow E, Mandelkow E M. Microtubules and microtubuleassociated proteins. Curr Opin Biol Cell. 1995;7(1):72-81.

57. Lahoz R, Hameroff S, Dayhoff J. Connection weights based on molecular mechanisms in Aplysia neuron synapses. Neurocomputing. 1996;11:179-202.

58. Mark M, Slaughter T, Moshiach S, Obrocka M, Fischer I. Tau is enriched on dynamic microtubules in the distal region of growing axons. J Neurosci. 1996;76(11):3601-19.

59. Fukada E, Ueda H, Rinaldi R. Piezoelectric and related properties of hydrated collagen. Biophys J. 1976;16(8):911-8.

60. Fröhlich $H$. Long range coherence and the actions of enzymes. Nature. 1970;228:1093.

61. Fröhlich $\mathrm{H}$. Longrange coherence and energy storage in biological. Systems. Int J Quant Chem. 1968;2:6419.

62. Georgiev D, Papaioanou N, Glazebrook J F. Solitonic effects of the local electromagnetic field on neuronal microtubules. Neuroquantology. 2007;5(3):276-91.

63. Trpisova B, Tuszynski J A. Possible link between guanosine 5 triphosphate hydrolysis and solitary waves in microtubules. Phys Rev Leett. 1997;53(3):3305-288.

64. Tuszynski J A, Trpisová B, Sept D, Sataric M V. The enigma of microtubules and their self-organizing behavior in the cytoskeleton. Biosystems. 1997;42(2-3):153-75.

65. Athenstaedt $\mathrm{H}$, Claussen $\mathrm{H}$. Evidence for pyroelectric and piezoelectric sensory mechanisms in the insect integument. Biophys J. 1981;35:365-74.

66. Tuszynski J A, Hameroff S, Sataric M, Trpisová V, Nip B. Ferroelectric behavior in microtubule dipole lattices: Implications for information processing, signaling and assembly/disassembly. J Theor Biol. 1995;174(4):371-80.

67. Roman F, Jaimes R, Osorio Y. Acercamiento al comportamiento dieléctrico de la procaína y sus soluciones: análisis de la permitividad dieléctrica con la procaina. Encuentro Internacional de Terapia Neural; Cuba 2004.

68. Martínez J, Ávila C, Fayad R. Transporte de señales eléctricas en microtúbulos: una aproximación cuántica. Rev Col Fis. 2010;42(2):1046.

69. Hameroff S, Nip A, Porter M, Tuszynski J. Conduction pathways in microtubules, biological quantum computation, and consciousness. Biosystems. 2002;42(64):149-68.

70. Bókkon I, Salari V, Tuszynski J A, Antal I. Estimation of the number of biophotons involved in the visual perception of single objectimage biophoton intensity can be considerably higher inside cells tan outside. J Photochem Photobiol B. 2010;100(3):100-6.

71. Amoroso R L. The Production of Frohlich and Bose-Elinstein Coherent States in vitro paracrystaline Oligomers Using Phase control laser Interferometry. Bioelectrochemistry. 1996;41:39-42.

72. Gauger E, Rieper E, Morton J, Vedral V. Sustained Quantum Coherence and Entanglement in the Avian Compass. Phys Rev Lett. 2011;106(4):4 páginas. 
73. Reimers J R, McKenzie R, Mark A, Hush N. Weak, strong, and coherent regimes of Fröhlich condensation and their applications to terahertz medicine and quantum consciousness. PNAS. 2009;106(11):4219-24.

74. Payán J C. La desobediencia vital. Buenos Aires: Salbe Ediciones;2005

75. Priel A, Ramos AJ, Tuszynski JA, Cantiello H.F. A biopolymer transistor: electrical amplification by microtubules. Biophys J. 2006;90(12):4639-43.
76. Priel A, Cantiello H, Tuszynski J A. A nonlinear cable-like model of amplified ionic wave propagation along microtubules. EPL (Europhysics Letters). 2008;83(6)

77. Etienne-Manneville S. From signaling pathways to microtubule dynamics: THE KEY PLAYERS. CURR OPIN CELL BIOL. 2010;22(1):104-11. 